\title{
LA VOZ DE GALICIA: ANA KIRO, RECEPCIÓN E IDENTIDAD NACIONAL
}

\section{CibránTenreiro Uzal}

Universidade de Santiago de Compostela, Dpto Ciencias da Comunicación

\section{Resumen}

Cuando la obra artística de una figura se identifica con una identidad nacional determinada, esta se convierte habitualmente en objeto de disputa y da lugar a lecturas en conflicto. Es el caso de Ana Kiro, cantante que desde los años setenta se convirtió en una figura enormemente popular en Galicia al abordar la experiencia de la emigración, el paisaje local o la cultura tradicional en canciones pop con elementos folclóricos. Durante años, Kiro fue a menudo interpretada (y criticada) como un icono conservador, pero en la última década han aparecido numerosas voces que buscan su resignificación como referente feminista, lusista o LGTBI. Este texto analiza la recepción de Ana Kiro y su conexión con los debates alrededor de la identidad nacional gallega.

Palabras clave: KIRO, ANA (1942-2010); RECEPCIÓN; IDENTIDAD NACIONAL; MÚSICA POPULAR; GALICIA

\section{GALICIA'S VOICE: ANA KIRO, RECEPTION AND NATIONAL IDENTITY}

\section{Abstract}

When an artist's work is identified with a particular national identity, the artist usually becomes an object of debate and gives room to antagonistic readings. That is the case of Ana Kiro, a singer who since the 1970s became an enormously popular figure in Galicia by dealing with the experience of emigration, local landscape or traditional culture in pop songs with folk elements. For years, Kiro was often interpreted (and criticized) as a conservative icon, but many voices have appeared during the last decade looking for her resignification as a feminist, lusist or LGTBI referent. This text analyses Ana Kiro's reception and its connection with the debates around Galician national identity.

Keywords: KIRO, ANA (1942-2010); RECEPTION; NATIONAL IDENTITY; POPULAR MUSIC; GALICIA

\footnotetext{
Tenreiro Uzal, Cibrán. 2021. “La voz de Galicia: Ana Kiro, recepción e identidad nacional". AusArt 9 (1): 69-83. D0I: 10.1387/ausart.22601
}

\section{AUSART}




\section{INTRODUCCIÓN}

Ana Kiro (1942-2010) es una de las figuras más populares en la historia de la música popular gallega, pero su celebridad es, precisamente, de carácter eminentemente local. No es un fenómeno único: Rebecca Williams y Ruth McElroy han utilizado el concepto de 'localebrity' (McElroy \& Williams 2010; Williams 2015) para analizar personalidades que, a diferencia de las figuras musicales, cinematográficas, deportivas o televisivas de fama internacional o estatal que centran habitualmente los estudios alrededor de la celebridad y su recepción, son conocidas en un contexto más reducido. En el caso de las citadas investigaciones de Williams y McElroy, ese contexto era el de Gales, una 'small nation' (Williams 2015, 2) cuya identidad nacional es personificada por estrellas mundialmente reconocidas como Tom Jones o Anthony Hopkins, pero también por personas desconocidas fuera de sus fronteras: "celebrity, place, and national identity also operate on a more local level where celebrities are dependent on both the media of the small nation or local area such as BBC Wales' programming (...) or local newspapers" (Williams 2015, 9).

En el caso de Ana Kiro, el hecho de que ese conocimiento sea limitado a Galicia (otra 'small nation') puede deberse, de manera similar a lo que sucede en Gales, a la atención prestada a su figura por los medios gallegos (especialmente la Televisión de Galicia, como se puede intuír de las numerosas referencias recuperadas desde ese medio en este artículo). No obstante, también es probable que se deba a su conexión con la identidad nacional gallega, tanto en su trayectoria artística como en la recepción que el público ha hecho de su obra y su figura, quizás en línea por lo apuntado por José Colmeiro: "In a stateless nation such as Galicia it has been predominantly in the cultural arena, rather than in the political front, that Galician identity has found its main channels of expression" (Colmeiro 2017, 5). En este artículo intentaremos, precisamente, crear un punto de entrada al rol que esa identidad ha jugado en la recepción de Ana Kiro y cómo ha ido evolucionando con el tiempo, especialmente en la década transcurrida desde su muerte.

Con esa intención, pondremos en relación una muestra de visiones de Ana Kiro que la relacionan con la identidad gallega, expresadas desde diferentes espacios, medios y puntos de vista, desde las redes sociales a la prensa convencional o nuevas obras artísticas. De esta manera tenemos en cuenta, como hizo Kirsty Hooper (2009) al explorar la relación de "Un canto a Galicia" de Julio Iglesias con la identidad nacional gallega observando los comentarios en YouTube alrededor de esta canción, el "historical shift of the public 
sphere from the institutional realm to the new communication space" (Castells 2007, 238). En ese sentido, parte del debate público alrededor de la artista es trasladado a través del contenido de tuits encontrados mediante la búsqueda de los términos "Ana Kiro" y "\#AnaKiro" en Twitter, así como los comentarios en los cinco vídeos de YouTube con un mayor recuento de reproducciones al buscar "Ana Kiro" o los comentarios en la página de Facebook "Fans Ana Kiro"1, siguiendo la línea de estudios como el citado de Hooper, pero también otros centrados en el fandom como el de Megan M. Wood y Linda Baughman (2012) sobre las prácticas de fans en Twitter alrededor de la serie Glee o el de Lucy Bennett (2011) sobre el comportamiento de grupos de fans de R.E.M. en la comunidad online Murmurs.com. Al mismo tiempo, Williams $(2015,10)$ señalaba el peso de la 'limited media ecology' de Gales a la hora de configurar la recepción de las celebridades locales, por lo que completaremos esta aproximación a la recepción de Ana Kiro observando la visión que se da de ella en los medios, especialmente aquellos más orientados al público gallego. En paralelo, intentaremos ubicar el contraste entre diferentes posiciones que se observa en la recepción de Ana Kiro en el marco de discusiones más amplias alrededor de la identidad gallega estudiadas desde el ámbito de los Galician Studies, así como apuntar los trazos más significativos de su figura para facilitar la comprensión del texto y abrir la posibilidad de futuros textos que estudien la recepción de esta artista de manera más pormenorizada o desde enfoques más ligados a la musicología o la sociología.

\section{ANA KIRO, LA GALLEGA}

Dada la naturaleza de este artículo, en lugar de arrancar el análisis con una breve nota biográfica sobre Ana Kiro que explique quién es al público no familiarizado con la artista, utilizaremos las definiciones y descripciones de ella que aparecen en parte de los contenidos analizados para acercarnos a su figura e ir viendo su conexión con la identidad gallega.

Por ejemplo, el bloguero Darth publicaba tras la muerte de la cantante un texto sobre ella y explicaba a sus 'lectores y colegas no gallegos' que, de algún modo "la muerte de Ana Kiro viene a ser en Galicia lo que la muerte de Lola Flores en España o la de Amália Rodrígues en Portugal" (2010). No es difícil encontrar paralelismos entre Kiro y Flores, ya que ambas utilizaron elementos de sus tradiciones de un modo no purista y destacaron por su dominio del 
humor popular. Con todo, quizás es más relevante la comparación con Amália Rodrigues, al tratarse también en su caso de una figura muy identificada con la identidad nacional de su país, hasta el punto de que es posible encontrar referencias a ella denominándola como 'la voz de Portugal' (García 1999). En el caso de Ana Kiro, un vistazo a cómo el público se refiere a ella en los medios o las redes sociales nos permite observar una insistencia en la comparación con figuras icónicas de otros lugares y en el subrayado de su carácter gallego. En ocasiones este aparece como adjetivo a añadir después de esa otra figura, para hablar de la "Dolly Parton gallega" (tweet de FanecasA30, 2020), la "Gloria Gaynor gallega" (APT, 2015), etc. En otras, se le menciona desde una idea colectiva de pertenencia y se convierte en "nuestra Tina Turner" (tweet de TRASHtero, 2017), "nuestra Rocío Jurado" (Bulnes, 2017), "nuestra Karina" (Gorkinha, 2020) y hasta se le da la vuelta a la cuestión para hablar de Oprah Winfrey como la "Ana Kiro americana" (tweet de Seriologa, 2018).

Estas comparaciones no son arbitrarias. Ana Kiro comenzó su carrera como cantante en Barcelona en los años sesenta, realizando sus primeras grabaciones para el sello Belter, en un estilo ye-yé similar al de cantantes como la citada Karina o Salomé. Con Dolly Parton comparte, por ejemplo, el rol que juegan sus orígenes rurales o la reivindicación de las mujeres trabajadoras en canciones destacadas de su repertorio (con el paralelismo entre canciones de Parton como "My Tennessee Mountain Home" y "9 to 5" y "A miña aldea" o "Galega traballadora" de Kiro). Si "I Will Survive" de Gloria Gaynor ha sido reivindicado en las celebraciones del orgullo LGTBQ (Skolnik 2018), lo mismo ha ocurrido con "Pídechas o corpo" (Radio Neria, 29 junio 2011), y a finales de los 90 y comienzos de los 2000 Ana Kiro presentó con gran éxito el talk show Tardes con Ana en la Televisión de Galicia², igual que Oprah Winfrey con otro programa que lleva su nombre (lo que explica el chiste de la usuaria seriologa antes citado). Esto podría indicar el carácter polifacético de la artista a lo largo de su carrera, pero también refleja en cierto modo la naturaleza mítica de los textos fan que indica Cornel Sandvoss: "facts become relative within the meta-narrative of the myth, which in turn is reflective of the fan's values, beliefs and image of self' (Sandvoss 2005, 134). Si el público utiliza los hechos para construir su propia interpretación de sus ídolos, parece que es habitual que las interpretaciones que se dan de Ana Kiro tengan en común apelar a la galleguidad.

¿Por qué sucede esto? Probablemente porque Galicia ha ocupado un lugar central en el discurso de Ana Kiro. En 1973, todavía afincada en Barcelona, convenció a su sello para grabar una canción en su lengua materna, "Galicia 
terra meiga", destacada a menudo como un hito en la prensa gallega por haber vendido 100.000 cintas, demostrando a Belter la viabilidad comercial de la música en gallego ante su inicial reticencia (Romero 2010; Varela 2010) ${ }^{3}$. A partir de ese momento, Kiro pasó a incorporar formas, repertorio e instrumentación tradicionales en canciones que a menudo conservaban la diversidad de influencias de su etapa previa ${ }^{4}$, participando de un estilo que ha sido descrito como "canción ligera gallega" (Pérez Pereiro 1999), "copla norteña pop" (Pereira 2010) o "canción popular gallega" (Campos Calvo-Sotelo 2009, 685). En el ámbito lírico, el gallego se convirtió en la lengua principal de sus grabaciones y las temáticas gallegas (especialmente la emigración) se convirtieron en centrales en sus letras ${ }^{5}$. La iconografía gallega pasó también a ser importante en su manera de presentarse al público ${ }^{6} \mathrm{y}$, especialmente, ese público la adoptó rápidamente: Ana Kiro llegaba a realizar entre 60 y 70 fechas en verbenas durante los meses de verano y en sus giras internacionales su éxito entre la diáspora gallega fue enorme, como muestra el hecho de haber actuado para 24.000 personas en Valle Fresco, una de las sedes de la Hermandad Gallega de Venezuela. Sus canciones apelan a su propia condición de emigrante ("Unha emigrante máis") y a los vínculos entre Galicia y los países latinoamericanos a través de esa emigración ("Galicia na Arxentina", "Galegos en México"), además de reflejar lo que parece una voluntad de superar los localismos, al mencionar habitualmente topónimos de puntos muy diversos de Galicia?.

\section{UNA FIGURA CONTROVERTIDA}

Teniendo en cuenta las características mencionadas anteriormente, es posible comprender que Ana Kiro es una figura muy popular en Galicia. Con todo, esa 'popularidad' es también la que hace que a lo largo de su carrera haya sido una figura controvertida, especialmente antes de su muerte. Las críticas a Ana Kiro han venido normalmente desde los ámbitos del nacionalismo gallego y la intelectualidad (normalmente también nacionalista), que la han rechazado señalando en ella un carácter conservador y su vínculo cultural y político con los gobiernos de Manuel Fraga ${ }^{8}$. Por ejemplo, un texto anónimo en la sección "A aldea global" del semanario nacionalista A Nosa Terra criticaba su presencia habitual en la TVG, y vinculaba su música al "estilo folclorista" de la sección femenina del franquismo (A Nosa Terra 1999). En 2010, un artículo en el digital Galicia Confidencial avanzaba una reivindicación de algunos aspec- 
tos de su figura pero la señalaba igualmente como "artista subvencionada" a sueldo del "fraguismo ${ }^{9}$ audiovisual" del canal autonómico gallego (Ayán 2010). Su popularidad también servía para utilizarla como instrumento para atacar otras obras, como en una crítica del poemario Fisteus era un mundo de Lupe Gómez que lo despreciaba tildándolo de "ruralismo simplista e almibarado ao máis burdo estilo Ana Kiro" (Pedreira 2001). Incluso, en otra línea, es posible encontrar textos que hablan de ella positivamente pero lo hacen con un tono de disculpa, como el texto del escritor y editor Francisco Castro en el que celebra su compromiso con la lengua gallega pero arranca con un "Obviamente, os meus gustos musicais están moi afastados dos temas de Ana Kiro" (Castro 2010). Entre estas muestras de rechazo, crítica o distanciamiento destaca un artículo de Jacinto Tasende en Diário Liberdade, que recoge cómo se presentó a Kiro en los medios después de su muerte y señala que funciona como un icono para perpetuar el estatus político e institucional de Galicia como un lugar "entranhável" y practicante de un galleguismo "doce" y "sem estridências", desligado de cualquier reivindicación (Tasende 2010). En contraste a estos ejemplos, el Partido Popular de Galicia no ha tenido problema en celebrar su figura, como señalaba una noticia de Europa Press: "los populares disfrutaron de un menú de empanada, pulpo y carne 'ao caldeiro' por 20 euros en un acto en el que se homenajeó a Ana Kiro" (25 sept. 2010).

¿Existe entonces una conexión entre el discurso de Ana Kiro y las políticas de Manuel Fraga? Podría ser. Joseba Gabilondo señaló cómo Fraga definió Galicia como una comunidad nacional diferenciada a la vez que vaciaba esa diferencia de contenido político, "endowing it with a strong affective content that he drew basically from Ramón Piñeiro's elaboration of morriña and saudade" (Gabilondo 2017, 169-70), una estrategia similar a la que Kirsty Hooper identificaba en el discurso de "Un canto a Galicia" de Julio Iglesias (Hooper 2009). Marta Pérez Pereiro situó esa canción de Iglesias como antecedente del estilo de Kiro en un trabajo que analizaba una muestra de sus letras para buscar los tópicos más repetidos y encontrar su ideología subyacente. Entre las estrategias líricas, destacaba la presentación de Galicia, de nuevo, como una tierra diferente de todas las demás, apoyándose en la lengua, el paisaje o la emigración como elementos distintivos, pero con una perspectiva sentimental y no política:

The point of view of Ana Kiro related to this social problem is pessimistic and does not include any kind of reaction or attempt of explanation of social and political causes of the emigration process. The themes are the returning to the land and its problems, 
the holidays spent at home and the relationship of Galicia with the countries that offered help to its emigrants.

(Pérez Pereiro 1999)

Precisamente, la autora compara esa actitud resignada de Ana Kiro con la de grupos como Fuxan os Ventos, que iniciaron su trayectoria en los años setenta y trabajaban con elementos de la música tradicional desde un discurso que abordaba en ocasiones las mismas temáticas pero, en su caso, identificaba causas, invitaba a la rebeldía o se significaba en conflictos políticos y luchas populares como la de As Encrobas ${ }^{10}$ (Souto 2016). El carácter conservador de la cantante puede ser entonces cierto en los aspectos señalados por Pérez Pereiro, pero señalarlo como el único llevaría a obviar las excepciones en su amplia discografía, el carácter político del hecho de cantar en gallego en su contexto $^{11}$, o el papel de la recepción de su figura. Como señalaba Sandvoss, el público refleja sus valores, sus creencias y su autoimagen al interpretar los textos, y en los últimos años la lectura de Ana Kiro como icono conservador ha ido dando paso a una serie de resignificaciones que pueden ejemplificar una tendencia que Helena Miguélez Carballeira y Kirsty Hooper han señalado en el debate alrededor de la cultura gallega, apuntando que este se sitúa cada vez más al margen de los foros e identidades institucionales para incorporar otros ejes (como el de clase o el de género) a la hora de tratar la identificación nacional (Miguélez Carballeira \& Hooper 2009).

\section{LO VIEJO Y LO NUEV0: (RE) SIGNIFICANDO A ANA KIRO}

La contraposición entre las críticas a Ana Kiro desde la órbita nacionalista y su reivindicación por parte del PP podría poner de manifiesto una cuestión señalada por Carlos Calvo Varela, que indicó que el galleguismo tiene un componente culturalista que Ilega a ser paradójico por su carácter elitista:

(...) como vai aspirar à hegemonia, se à sua vez se apresenta como um modo de distinção? Eis uma nação que as elites escrevem. Uma e outra vez, cederam-se aos contrarrevolucionários do "galeguismo de lacão com grelos" espaços maçiços em que se 
efetua a reprodução social: dos estádios de futebol à cozinha, passando pola música pop em galego.

(Calvo Varela 2015, 43)

En ese sentido, el carácter eminentemente popular de Kiro permite que siga siendo pensada en relación a ese galleguismo 'de lacón con grelos', aparentemente despolitizado o sentimental y promovido por el fraguismo. Con todo, numerosas personas, normalmente más jóvenes, han ido empezando a reivindicarla desde perspectivas más explícitamente políticas, alejándose del elitismo desde el que era recibida en vida.

En la primera línea, más ligada a esa visión sentimental de Galicia señalada por Pérez Pereiro en su artículo o al contenido afectivo que Gabilondo identifica en el fraguismo, la página "Fans Ana Kiro" en Facebook recoge una serie de comentarios que vinculan a Ana Kiro con Galicia insistiendo en el factor de la emigración o identificándola con la tierra: "Era unha gran voz que representaba moi ben a nosa terra" (comentario de Rey Couto, 2020), "Cantos recordos de ela e cantas lagrimas chorei eu solo con oila cantar cando estaba en bilbao tiña morriña de ela" (comentario de Pampin, 2020). El relato que habla de la emoción de escuchar sus canciones en la diáspora es habitual. En un programa de la TVG alrededor de la citada canción "Galicia terra meiga" se entrevistaba a personas anónimas sobre su relación con Ana Kiro y aparecían opiniones similares, como la de un hombre que, preguntado por lo que pensaba al escuchar a Ana Kiro estando en Australia, respondía "Home, pois na terra dun. Fíxonos chorar moitas lágrimas as cancións dela" ("Galicia, terra meiga (Ana Kiro)", 22 ene. 2013).

Los comentarios en los vídeos de sus canciones más reproducidas en YouTube indican algo parecido, pero aparecen algunos que indican una visión más próxima al nacionalismo gallego organizado, afirmando Galicia como país y señalando la oposición de Ana Kiro al franquismo (Den27, comentario al vídeo de Youtube "Vivir na Coruña"). En una entrevista personal, el administrador de la citada página de Facebook identificaba precisamente a Kiro como una artista "de canción protesta", y en lugar de oponerla a figuras como Fuxan os Ventos la comparaba con otro de sus músicos favoritos, Suso Vaamonde (que estuvo en la cárcel por injurias a la patria):

Cantaban os mesmos problemas, porque eran os problemas que tiñan no país daquela. O que pasa é que Ana tiña un enganche especial, que ao mellor Suso Vaamonde, que tamén tiña moito público, non o colleu tanto como Ana. Sobre todo iso, porque eran 
canción protesta, que era o que naquel momento máis triunfaba no país ${ }^{12}$.

En este sentido, se observa cómo la contraposición entre las figuras vinculadas al nacionalismo más politizado y figuras de éxito más transversal, presente como vimos alrededor de Ana Kiro y de artistas como Juan Pardo ${ }^{13}$, no siempre es percibida como tal: es posible pensar a Ana Kiro sin vincularla al fraguismo. En Twitter se encuentran referencias a pintadas que la identificaban como 'patriota galega' (término que desde el nacionalismo se aplica a iconos como Castelao) (tweet de Maruxinha3, 2010) o se destacan canciones como "Sempre en Galicia" o "Galicia ceibe" (tweet de Vivaantes, 2013), que funcionan como excepciones a la resignación y animan a la lucha contra los caciques o afirman la condición de esclavos de los gallegos.

Ha habido más casos en los que se selecciona una parte del repertorio o la trayectoria de la cantante para aplicarla a un discurso ideologizado y ajeno al galleguismo de Fraga. Por ejemplo, el lusismo (una corriente que considera que el gallego es la misma lengua que el portugués y sus variantes y defiende una confluencia normativa con estas) ha utilizado a menudo su canción "Se vas ao Brasil" como ejemplo de sus tesis: "É um ícone demasiado grande como para resumir nada num tweet, mas queremos lembrar hoje aquela cançom dela que dizia: 'Se vás ao Brasil ou a Portugal verás, meu amigo, que grande e fermoso é o nosso falar'" (tweet de Emgalego, 2010). También se ha reivindicado el carácter de pionera feminista de Ana Kiro (Bonmatweet 2019), eludiendo parte de su representación de las relaciones de pareja y destacando letras como la de la mencionada "Galega traballadora" (que visibiliza la carga de trabajo doméstico sumada a la del trabajo asalariado) o el control que ejerció sobre su carrera y el hecho de haberse separado de su marido en pleno franquismo. Como avanzábamos al inicio, también ha sido tratada como icono LGTBI cada vez con más frecuencia, especialmente alrededor de la citada canción "Pídechas o corpo" (que se interpreta como una reivindicación de la libertad sexual), de la presencia habitual del travestismo en sus espectáculos o del hecho de que donase sus pelucas a drag queens. De hecho, la "drag pandeireteira" Meiga-i menciona a Kiro como la gran diva pop gallega y una de sus principales influencias ("Coñecemos a Meiga-I, a primeira 'drag pandeireteira'“, 31 jul. 2020).

En esa línea, la resignificación de Ana Kiro por parte de la gente más joven podría conectar también con la tendencia contemporánea a aproximarse a músicas populares que hasta hace poco no gozaban de capital cultural ${ }^{14}$. 
Un ejemplo claro estaría en "Muinheira de interior", el tema del grupo de trap gallego Boyanka Kostova: en él recuperan la letra de dos temas de Kiro ("Arrastráche-lo cu polas pallas" y "Unha noite no muíño") y su tono de humor costumbrista en un contexto musical diferente, más electrónico y agresivo. Precisamente, el youtuber Olaxonmario (2018) ha ironizado sobre el parecido entre las estrategias de Rosalía como "apropiadora cultural" y las utilizadas por Ana Kiro (que mezclaba rumbas, merengues y pasodobles con gaitas y panderetas) y el también youtuber Aquel-e la ha denominado como "icono trash" gallego (2018).

En cualquier caso, como se puede ver, todas estas resignificaciones pasan por su carácter gallego. Una de las comparaciones iniciales, que se refería a Ana Kiro como "nuestra Tina Turner", la hizo el escritor y músico Antón Reixa, explicando que la cultura gallega es una cultura "de muchas carencias" y que Kiro jugó el papel de referencia local en la música ligera. Diez años después de su muerte, observamos cómo las carencias que cubre no están solo en el ámbito musical, sino en los debates culturales en general, donde funciona (en su rol de "localebrity") como un referente transversal que diferentes comunidades reivindican como propio dentro de Galicia ${ }^{15}$. En ese sentido, a pesar de que el discurso y la trayectoria de Ana Kiro tengan un componente conservador, la recepción (seguramente ayudada también por la distancia temporal y la benevolencia provocada por su muerte temprana a causa de un cáncer) ha ido separándose de lecturas más elitistas para hacer emerger a través de ella discursos que abordan la identidad gallega en relación al feminismo, el movimiento LGTBIQ o la revalorización de la cultura popular, conectando la capacidad del fandom para crear significados con el desplazamiento del debate identitario desde los espacios institucionales a otros (como señalaban Miguélez Carballeira y Hooper).

\section{Referencias bibliográficas}

Ayán Vila, Xurxo Miguel. 2010. "Kírote". Galicia Confidencial, 17 ago. http://emprende.galiciaconfidencial.com/noticia/6195-kirote

Bennett, Lucy. 2011 "Music fandom online: R.E.M. fans in pursuit of the ultimate first listen". New Media \& Society 14(5): 748-63

Bulnes Fraga, Ana. 2017. "16 canciones que debes escuchar antes de venir a Galicia". Matador Network. 2 jun. https://matadornetwork.com/es/16-canciones-que-debes-escuchar-antes-de-venir-galicia/

Calvo Varela, Carlos. 2015. Diários. Santiago de Compostela: Através 
Campos Calvo-Sotelo, Javier. 2009. "La música popular gallega en los años de la transición política (1975-1982)". Tesis Univ. Complutense de Madrid

Castells Oliván, Manuel. 2007. "Communication, power and counter-power in the Network Society". International Journal of Communication 1: 238-66

Castro Veloso, Francisco. 2010. "Ana Kiro". Franciscocastro.gal (web personal del autor), 13 ago. https://franciscocastro.gal/ana-kiro/

Colmeiro, José F. 2017. Peripheral visions/global sounds. From Galicia to the world. Liverpool: Liverpool University

Fernández Rego, Fernando. 2019. Unha historia da música en Galicia: 1952-2018. Vigo: Galaxia

Gabilondo Alberdi, Joseba. 2017. "The master signifier of Galician culture: Manuel Fraga and undemocratic, affective populism". En Rerouting Galician Studies: Multidisciplinary interventions, edited by Benita Sampedro Vizcaya \& José A. Losada Montero, 165-78. London: Palgrave Macmillan

García, Javier. 1999. "Muere en Lisboa, a los 79 años, Amália Rodrigues, la voz más profunda del fado". El País, 7 oct. https://elpais.com/diario/1999/10/07/cultura/939247201_850215. $\mathrm{html}$

Hooper, Kirsty. 2009. "The many faces of Julio Iglesias: "Un canto a Galicia", emigration and the network society". Journal of Spanish Cultural Studies 10 (2): 149-66

Lama López, María Xesús. 2017. Rosalía de Castro: Cantos de independencia e liberdade (1837-1863). Vigo: Galaxia

Lorenzo Danta, Serafín. 2014. "El día que nació el fraguismo". La Voz de Galicia. 17 dic. https://www.lavozdegalicia.es/noticia/galicia/2014/12/17/dia-nacio-fraguismo/0003_201412G17P7991.htm

Macías Blanco, Alejandro. 2018. "20 años de 'Tardes con Ana' en TVG”. Carta de Ajuste. 25 abril. http://carta-de-ajuste.blogspot.com/2018/04/20-anos-de-tardes-con-ana-en-tvg.html

Mc Elroy, Ruth \& Rebecca Williams. 2010. "Remembering ourselves, viewing the others: Historical reality television and celebrity in the small nation". Television \& New Media 12 (3): 187-206

Miguélez Carballeira, Helena \& Kirsty Hooper. 2009. "Introduction: Cricital approaches to the nation in Galician studies". The Bulletin of Hispanic Studies 86(2):201-12

Olaxonmario. 2018. "Ana Kiro apropiadora cultural". Vídeo de Youtube, 4:15. 16 oct. https:// www.youtube.com/watch?v=nf89LiqxZMo

Pedreira Rodríguez, Penélope. 2001. "Un ruralismo simplista”. O Ximnasio de Academo (web)

Pereira Revuelta, Nonito. 2010. "Ana Kiro, la reina”. El Ideal Gallego, 25 sept.

Pérez Pereiro, Marta. 1999. "A rumba da gaita: A sociological approach to the music of Ana Kiro". Trabajo de grado. Univ. of South Wales

Skolnik, Jes. 2018. "50 songs that define the last 50 years of LGBTQ+ Pride". Pitchfork.com, 18 jun. https://pitchfork.com/features/lists-and-guides/50-songs-that-define-the-last-50-yearsof-lgbtq-pride/

Romero García, Santiago. 2010. "La banda sonora de la emigración”. Faro de Vigo. 25 sept. https://www.farodevigo.es/sociedad/2010/09/25/banda-sonora-emigracion-17814495.html 
Sánchez Porta, Juan (Oro Jondo). 2021. Dame más gasolina: Un recorrido por la música de gasolinera. Barcelona: Cúpula

Sandvoss, Cornel. 2005. Fans: The mirror of consumption. Cambridge: Polity

Souto Eiroa, Xurxo Manuel. 2016. "Unha canción marabillosa, galega e panteísta”. Boletín da Real Academia Galega 377: 169-177

Tasende, Jacinto. 2010. "Ana Kiro, funcionalidade político-ideológica dum ícone”. Liberdade. 25 sept. https://www.diarioliberdade.org/galiza/cultura-m\%C3\%BAsica/7008-ana-kiro-funcionalidade-politico-ideologica-dum-icone.html

Varela, Ángel. 2010. “La gallega que superó a Julio Iglesias”. La Voz de Galicia. 25 sept. https://www.lavozdegalicia.es/noticia/cultura/2010/09/25/gallega-supero-julio-iglesias/00031285368018153500840.htm

Williams, Rebecca. 2015. "Localebrities, adopted residents, and local characters: audience and celebrity in a small nation". Celebrity Studies 7(2): 154-68.

Wood, Megan M. \& Linda Baughman. 2012. "Glee fandom and Twitter: Something new, or more of the same old thing". Communication Studies 63 (3): 328-44

Otras fuentes

"A noite de Ana", especial TVG. Publicado por O, o., 'Galiza Trash'. Vídeo de Youtube, 21 feb. 2018 https://youtu.be/Rol6AD-O6AY

"A aldea global". A Nosa Terra, 1 jul. 1999

"Ana Kiro na Radio Galega IV". Emitido 24 sept. 2011. Podcast de radio Corporación Radio e Televisión de Galicia, 17:59. http://www.crtvg.es/cultural/especiais/radio-galega-ana-kiro-na-radio-galega-iv

"Ana Kiro vendeu 100.000 cintas coa súa primeira canción en galego, 'Galicia Terra Meiga!'“. Corporación Radio e Televisión de Galicia, programa emitido 25 abril 2018, 10:28. https:// www.crtvg.es/tvg/a-carta/ana-kiro-vendeu-100-000-cintas-coa-sua-primeira-cancion-engalego-galicia-terra-meiga

"Ana Kiro, toda unha vida", programa especial que TVG emitiu ao día seguinte da morte da artista. Publicado por TRASHtero. Vídeo de Youtube, 1:12:33., 19 sept. 2017. https://youtu. be/xHqV1Ys2DYM?t=1548

"Cafetería Ana Kiro". Aún Pillas Tortillas! (blog). Publicada por APT, 6 marzo 2015. https://aunpiIlastortillas.com/cafeteria-ana-kiro/

"Coñecemos a Meiga-I, a primeira 'drag pandeireteira'“. Programa emitido 31 julio 2020. Vídeo Corporación Radio e Televisión de Galicia, 04:22. http://www.crtvg.es/tvg/a-carta/conecemos-a-meiga-i-a-primeira-drag-pandeireteira

"Feijóo erige a Silleda en el Rodiezmo del PPdeG y llama a los suyos a luchar contra "315 Zapateros" del PSdeG". Europa Press.es, 25 sept. 2010. https://m.europapress.es/galicia/ noticia-feijoo-erige-silleda-rodiezmo-ppdeg-llama-suyos-luchar-contra-315-zapateros-psdeg-20100925203119.html

"Galicia, terra meiga (Ana Kiro)". Programa emitido 22 ene. 2013. Vídeo Corporación Radio e Televisión de Galicia, 30:14. https://www.crtvg.es/tvg/a-carta/galicia-terra-meiga-ana-kiro

"Manifesto dobarrista", 2 CD-ROM (30 canciones), tributo a Andrés do Barro. Regueifa Plataforma, Falcatruada \& Lonxa Cultural, 2007 
"Morreu Ana Kiro". Hace no mucho tiempo, en una galaxia no muy lejana... (blog). Publicado por Darth, 24 sept. 2010. http://endevewars.blogspot.com/2010/09/morreu-ana-kiro.html

"Ribeira celebrará o Día do Orgullo reivindicando o 'Pídechas o corpo' como himno gay galego". Radiofusion.gal. Publicada por Radio Neria, 29 xuño 2011. https://www.radiofusion.gal/actualidade/novas/ribeira-celebrar\%c3\%a1-o-d\%c3\%ada-do-orgullo-reivindicando-o- $\%$ e $2 \% 80 \% 9$ cp $\%$ c3\%addechas-o-corpo\%e2\%80\%9d-como-himno

"Vivir na Coruña". Canción de Ana Kiro, 1983. Publicado por Den27. Vídeo de Youtube, 2:54, 7 feb. 2010. https://www.youtube.com/watch?v=mkNsJoHOUjQ

\section{Facebook}

Pampin, Angelines. "Hay una fecha marcada en nuestro calendario...". Fans Ana Kiro. Facebook, 24 ene. 2020. https://www.facebook.com/772207509467769/photos/a.772581512763702/2887694574585708/

Rey Couto, María Teresa. "Bien es cierto que llevamos...". Fans Ana Kiro. Facebook, 29 jul. 2020. https://www.facebook.com/772207509467769/photos/a.772581512763702/3347545348600626/

Twitter

Bonmatweet. "Agora que para dignificar a obra dunha muller". Twitter, 7 marzo 2019. https:// twitter.com/Bonmatweet/status/1103658925910564865

Emgalego. "Hoje fam-se 10 anos do falecimento de Ana Kiro". Twitter, 24 sept. 2020. https:// twitter.com/emgalego/status/1309093682709254145

FanecasA30. “O @Snob chamabalhe a Dolly Parton galega”. Twitter, 22 ago. 2020. https://twitter.com/FanecasA30/status/1297110463059316736

Gorkinha. “Nuestra Karina!!!”. Twitter, 3 nov. 2020. https://twitter.com/gorkinha/status/1323711730015490049

Maruxinha3. "Ana Kiro patriota galega!" Twitter, 24 sept. 2020, https://twitter.com/maruxinha3/ status/1309213284659269632

Seriologa. "A Ana Kiro americana é Oprah". Twitter, 25 ene. 2018. https://twitter.com/seriologa/ status/956459641764040705

Vivaantes. "Nom se pode ser patriota". Twitter, 7 jun. 2013, https://twitter.com/VIVAANTES/status/342940755586846721

\section{Notas}

${ }^{1}$ Estas búsquedas se llevaron a cabo en diciembre de 2020 de cara a elaborar una presentación con el título "La voz de Galicia: Ana Kiro, fandom e identidad nacional" para las Jornadas Kantatzen Duten Herriak 2020 de la Universidad del País Vasco, expuesta el 11 de diciembre del mismo año.

${ }^{2}$ El programa, producido por Gestmusic, tuvo un share del $18 \%$ en su primera temporada y se mantuvo en antena durante cuatro años (Macías 2018).

${ }^{3}$ En una entrevista personal a Anxo Lamas, responsable de la página de Facebook "Fans Ana Kiro", del 12 de diciembre de 2019, indicaba que el acceso a los discos de Ana Kiro se 
daba especialmente a través de la distribución en gasolineras, algo que conecta en cierta manera con el auge de las estaciones de servicio como punto de venta musical desde principios de los setenta, señalado por Juan Sánchez Porta. Este autor liga esa tendencia en la distribución con diversos géneros y figuras musicales de éxito entre las clases populares pero con poco prestigio crítico y apoyo mediático, identificables bajo el término "música de gasolinera" (2020: 18-19).

${ }^{4}$ Entre esas formas destacan la muiñeira en canciones como "Unha noite no muíño" o la jota en "Carta d'un emigrante", identificadas como tal en los créditos de Ana Kiro (1980) y A miña aldea (1979). Lo referido al repertorio se observa en discos como Arrastráche-lo cu polas pallas (1989), donde hasta 7 de los 10 temas son adaptaciones de canciones populares gallegas, y la incorporación de instrumentos como la gaita o la pandereta se traslada también a títulos de canciones como "A rumba da gaita" o "Pandeirada galega". De manera significativa, Javier Campos Calvo-Sotelo (2009, 684-686) señala cómo Kiro, Andrés do Barro, Juan Pardo o María Ostiz adaptaron "el contenido y sonoridad de sus producciones" a esa denominada "canción popular gallega" en función de una nueva demanda generada alrededor de grupos como A Roda "a partir de un sustrato que proviene, efectivamente, del repertorio más genuinamente popular, que se recrea en un estilo lúdico, levemente hedonista, próximo en grado variable a las reivindicaciones más comunes de la entente social-galleguista y muy efectivo en relación a las ventas". Esto implica un contraste con las primeras grabaciones y actuaciones de los citados artistas, que el autor ubica dentro de un subtipo diferente de la "canción gallega": la "canción hispano-galaica". Esta categoría estaría próxima a "la llamada canción española y su icono referencial flamenquista -a la par que deudores de los rasgos más estereotipados de la canción ligera internacional de moda-, caracterizados por la finalidad comercial", y en ella operaría "un folklorismo insustancial y blando", alejado "de las fuentes de referencia del legado tradicional".

${ }^{5}$ Entre las 24 canciones que componen la muestra de su análisis de las letras de Ana Kiro, Pérez Pereiro (1999) señaló el sentimiento de ser gallego y la emigración, por ese orden, como los dos temas más habituales.

${ }^{6}$ La franja azul sobre fondo blanco de la bandera gallega aparece en las portadas de los álbumes Canta a Galicia (1977), Eu• Galicia (1982) o Ana Kiro (1987), mientras aparece ataviada con el traje tradicional en los álbumes Recordando a Galicia (1976), Galicia terra meiga (1977) o Ana Kiro (1978) y delante de la catedral de Santiago en el LP Galega traballadora (1982).

${ }^{7}$ Algunos ejemplos se ven directamente en los títulos: "Arzúa no camiño", "Vivir na Coruña", "Mazaricos", "Camariñas da alma", "Vou a Marín".

${ }^{8}$ Manuel Fraga presidió la Xunta de Galicia durante cuatro legislaturas (1990-2005), después de haber sido Ministro de Información y Turismo durante la dictadura franquista (19621969) y haber presidido tanto el Partido Popular como la formación de la que salió este, Alianza Popular.

${ }^{9}$ El término 'fraguismo' ha sido utilizado tanto en relación al período de gobierno de Fraga en Galicia (Lorenzo 2014) como en relación a su modelo político y cultural (Gabilondo 2017).

10 En 1977, las tierras de la parroquia de As Encrobas (Cerceda, A Coruña) fueron expropiadas para la explotación de lignito y la instalación de la central térmica de Meirama, dando lugar a una fuerte respuesta del movimiento agrario y el nacionalismo gallego.

${ }^{11}$ En esta línea, son significativas las declaraciones de Andrés do Barro recogidas en el interior del disco homenaje Manifesto Dobarrista (2007), que indican cómo en artistas normalmente desvinculados de los movimientos políticos organizados o la oposición al franquismo podía haber, por otra parte, una voluntad de lucha en cuestiones como el 
conflicto lingüístico, llevada a cabo precisamente al utilizar el gallego para tratar temáticas no explítamente políticas: “(...) na miña terra o galego está considerado un idioma para o proletariado. Eu pretendín, coas miñas cancións, loitar para que desapareza esta imaxe de menosprezo cara miña lingua. Quero demostrar que non é só un idioma para os humildes, e, así mesmo, que a xente humilde vexa que coa lingua que fala se poden cantar cousas alegres e de hoxe".

${ }^{12}$ Entrevista personal a Anxo Lamas, 12 de diciembre de 2019.

${ }^{13}$ En su Unha historia da música en Galicia: 1952-2018, Fernando Fernández Rego recoge las acusaciones de oportunista a Pardo después del éxito comercial de su disco en gallego Galicia, miña nai dos dous mares (1976), ejemplificadas por las declaraciones del músico Bibiano, miembro del colectivo Voces Ceibes, que identificaba a Pío Cabanillas (Ministro de Información y Turismo en 1974 y miembro de UCD posteriormente) y al periodista Antonio D. Olano como "ideólogos" de un álbum pensado "para aplacar os éxitos de Voces Ceibes", acusándolos de defender y distribuir "unha imaxe de Galicia histórica, nostálxica, sen problemas, costumista, rexional e morriñenta" (Fernández Rego 2019, 108).

${ }^{14}$ Es algo que se puede observar a través de las versiones o referencias que funcionan como guiño en la obra de artistas jóvenes: Rosalía ha versionado "Me quedo contigo" de Los Chunguitos, C.Tangana ha sampleado a Los Chichos o recuperado letras y melodías de Rosario Flores o Alejandro Sanz, Rojuu ha publicado "Love 2 Camela", y en Galicia Novedades Carminha colaboró con Esteban y Manuel en una versión de "A Santiago voy" de Los Tamara.

${ }^{15}$ Salvando las distancias (de época, prestigio o institucionalización), estas disputas pueden recordar a las que se dan alrededor de la figura más asociada a la idea de Galicia, Rosalía de Castro, de quien María Xesús Lama ha señalado la "tendencia dos biógrafos a atribuírlle unha personalidade que encaixe co ideal feminino de cada quen, sexa pura e muller dun só home, apaixonada ou revolucionaria" (Lama 2017). 